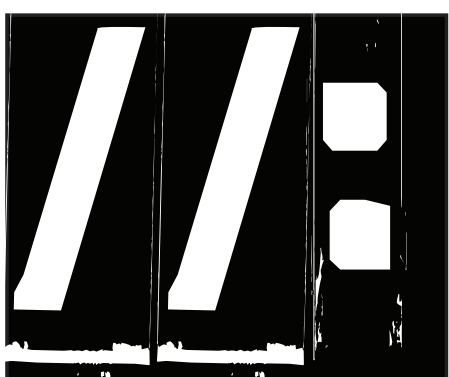

educa"ción y cōmunicación 14: 135-144 Mayo 2017

\title{
DIALOGÍA: UN DESAFÍO PERMANENTE PARA LOS PROFESORES EN CURSOS ONLINE
}

\section{Dialogue: a permanent challenge for teachers in online courses}

\section{José Lauro Martins}

Filósofo, doctor en Ciencia de la Educación. Profesor de la Universidad Federal de Tocantins. Palmas (Brasil)

E-mail: jlauro@uft.edu.br

\section{Resumen:}

Este trabajo es parte de una pesquisa en que proponemos evaluar la dialogía en los foros de estudios de un curso b-learning, motivada por la dificultad en orientar e incentivar los profesores a promover debate en los foros para que hubiese una dialogía capaz de construir una narrativa con contenidos y presencia. Se percibe, al final, que no basta la participación para que la dialogía acontezca, es preciso entender los límites y la calidad de la mediación, principalmente en las horas iniciales de lanzamiento de una discusión.

Palabras clave: Educación online; foros; dialogía; gestión del aprendizaje.

\section{Abstract:}

This work is a part of an inquiry in which we propose to evaluate the dialogía in the forums of studies of a course b-learning, motivated by the difficulty in orientating and stimulating the teachers to promote debate in the forums in order that there was a dialogía capable of constructing a narrative with contents and presence. There is perceived, ultimately, that the participation is not enough in order that the dialogía happens, it is necessary to understand the limits and the quality of the mediation, principally in the initial hours of launch of a discussion.

Key words: Education online; forums; dialogía; management of the learning.

Recibido: 31-01-2017 Revisado: 24-02-2017 Aceptado: 28-02-2017 Publicado: 01-05-2017

https://doi.org/10.25267/Hachetetepe.2017.v1.i14.12 


\section{II: Educación, Comunicación y Cultura Contemporánea}

\section{Introducción}

i

En qué estamos pensando cuando hablamos de in$\zeta$ teracción en los cursos online? Esta puede ser una pregunta retórica o un cuestionamiento fundamental cuando se planifica una buena calidad educativa en un curso mediado por las tecnologías de la Web. Puede parecer trivial hablar de la interacción en los cursos tradicionales en que profesores y aprendices tienen relativa proximidad. Aunque, la interacción sin un diálogo efectivo no contribuye significativamente para el aprendizaje. Sin embargo, habituamos a considerar que hay interacción por el simple hecho de poder encontrarnos en el aula tradicional o en otros espacios académicos

Si la intención es una fuerte interacción, es preciso invertir en la calidad de los discursos que efectuamos durante el proceso de aprendizaje. De esa manera, la distancia entre estudiantes y educadores puede ser minimizada o hasta eliminada si hay una percepción de presencia significativa en la gestación del aprendizaje (Martins, 2014). De esa forma, la distancia se desvanece entre los dedos de la estructura tecnológica que posibilita el diálogo permanente y confirma el concepto de "distancia transnacional" cuñado por Michel Moore.

\section{El diálogo en los foros online}

Considerando el foro virtual uno de los principales instrumento de comunicación y, por consiguiente, de interacción en los cursos online, debemos tener una mirada atenta a los "diálogos" en este instrumento más allá de favorecer una presencia social importante, estructurar una presencia pedagógica (Garrison,
Anderson y Archer, 2001) que soporte el proceso de aprendizaje. No es un cuidado ingenuo, sino un cuidado con fuerte presencia epistemológica. Pues las narrativas, bien como los discursos que subsidian el proceso formativo en el ambiente virtual precisan tener siempre un carácter dialógico, destinado para alguien. Aunque direccionemos nuestro discursos a uno o muchos discentes, es preciso que cada un perciba que ese argumento fue estructurado para él.

En muchos casos, el discurso descuidado tiende a un monólogo. Apenas una réplica en una narrativa que no presupone ninguna respuesta (Bakhtin, 1997: 285). No podemos decir que no hay alguna interacción en un discurso leído por alguien, aunque no lo incite de alguna forma a la aproximación entre los sujetos de la narrativa. Backhtin recuerda bien que "La relación dialógica tiene una amplitud mayor que el habla dialógico, en una acepción estricta. Incluso entre producciones verbales profundamente monológicas, se observa siempre una relación dialógica" (1997: 355) (1). Sin embargo, en vista de los objetivos de un foro de estudios, no basta una "charla dialógica", sino con una relación "dialógica".

Es preciso considerar que las narrativas en un foro de estudios tiene un contexto que va más allá de los contenidos del curso y de la tecnología empleada en la interacción. Como dice Scorsolini-Comin (2014: 252), "El producto del acto del habla, la enunciación, es de naturaleza social, determinada por la situación más inmediata o por el medio social más amplio". Por tanto, no se trata de un "discurso virtual", aunque haya sido utilizado por una tecnología que permitió la disposición en el ciberespacio, el discurso tuvo autoría, contexto e historia que fundamentaron la compresión y la interacción. 


\section{II: Educomunicación mestiza y en movimiento: 11. Desafios a un conocimiento multicultural}

Paulo Freire es un autor de gran relevancia para la discusión sobre los "diálogos" que se estructuran en el proceso educativo. Además de la comprensión backtiniana del discurso como instrumento de comunicación, precisamos de la sensibilidad freireana para entender el discurso como parte formativa y no solo como simple comunicación establecida en los foros de los cursos online. Freire (1979: s/p) dice al respecto: "El diálogo es el encuentro entre los hombres, mediatizados por el mundo, para designarlo". Comprendemos que este sea el diálogo que precisamos en los foros virtuales, principalmente por los formadores que tienen el papel de mediar el desvelamiento del mundo por medio del conocimiento. En este sentido, el diálogo es un instrumento que contribuye para "(...) transformar y humanizar, no puede reducirse a un depositario de ideas en otros. No puede también convertirse en un simple intercambio de ideas, para ser consumidas por los permutantes" (Freire, 1979: $\mathrm{s} / \mathrm{p}$ ). Por tanto, es preciso una presencia social permanentemente del mediador, con cuidado vigoroso con el discurso pedagógico y la frecuencia sistemática para que los aprendices se sientan solos, aunque participando de un grupo.

Otro aspecto importante a considerar es que no se puede culpar a la tecnología o al contenido por la dificultad de establecer un diálogo, como bien dice Paulo Freire, "El diálogo problematizador no depende del contenido que va a ser planteado. Todo puede ser debatido" (Freire, 1975). Además, es fundamental considerar la dialogía como parte metodológica de la formación, principalmente cuando se trata de la formación de educadores. La educación online precisa ser interactiva/colaborativa y cabe al profesor mediar lo que puede ser más importante para la formación, que el propio contenido. (Scorsolini-Comin, 2014: 257)

Otro aspecto muy importante del diálogo pedagógico es la necesidad de la autoría. A ejemplo de esto, podemos encontrar foros en una secuencia de mensajes que apenas repitan trechos de lecturas. Lo que daría un nivel muy bajo de dialogía. En este caso, el mediador precisa intervenir para que, de hecho, se establezca un diálogo y no una secuencia de mensajes. Aunque sea una manifestación simple en debates sobre cuestiones complejas, no ha diálogo sin autoría. En este aspecto, el diálogo se convierte en hilo conductor de la gestión del aprendizaje (Martins, 2014). La autoría en el diálogo no se da de forma mecánica simplemente, pues hay interlocutores que también son sujetos y pueden interferir en los contenidos y en el lenguaje. (Scorsolini-Comin, 2014: 261). Así, el diálogo puede ser una línea tenue entre la superación de la educación tradicional que refuerza la dependencia y la estructura de gestión del aprendizaje que refuerza la construcción de la autonomía. Colocado esto, la autonomía no es una concesión de los profesores a los aprendices, ni tampoco una exigencia de los cursos online, sino una parte significativa del currículo que precisa ser orientada y construida (Martins, 2014). Es preciso comprender que reforzar la consciencia de la autoría/autonomía no deslegitima el discurso científico; al contrario, reafirma la posición de la crítica y del diálogo que soporta el pensamiento científico. De cierta manera, deslegitima las formas tradicionales de la educación estructurada sobre el poder del conocimiento/profesor y del no saber/alumno. 


\section{IIA Educación, Comunicación y Cultura Contemporánea}

\section{La investigación}

Trajimos, para este artículo, parte de una pesquisa en que proponemos evaluar la dialogía en los foros de estudios en un curso de ambiente virtual y con encuentros presenciales bimestrales ofrecido por la Universidad Federal de Tocantins para cerca de 200 profesores de escuelas públicas (alumnos del curso) distribuidos en cinco grupos. El curso disponía de, por lo menos, un foro de estudios en cada grupo. Esta investigación fue motivada por la dificultad de orientar e incentivar a los profesores promover el debate para que hubiese una dilogía capaz de construir una narrativa. Levantamos dudas sobre lo que los profesores entendían por diálogo cuando se trataba de una clase online.

\section{La metodología}

Conducimos una investigación cualitativa (Flick, 2005) y analizamos las participaciones en los foros por medio de estrategias de análisis de contenido (Bardin, 2011). Cada mensaje en los foros estudiados fue una unidad de análisis y procuramos entender si había una narrativa en los instrumento o apenas una recopilación de mensajes. Fueron analizados 482 mensajes, siendo 96 mensajes de 5 profesores de curso, en la primera fase y ampliamos para 200 mensajes en la segunda fase, visando la estabilización de los datos. Los procedimientos tuvieron la siguiente secuencia: colecta y preparación de los datos, codificación de las unidades de análisis (cada mensaje), categorización e interpretación.

\section{Presentación y análisis de datos}

Consideramos dos aspectos estructurales en las narrativas dentro de los foros de los cursos virtuales: la participación y el contenido, bases obvias, sin embargo complejas, desde el punto de vista de la gestión del aprendizaje. Una vez que la situación ideal para la dialogía es que el deber de participar por exigencia académica venga a ser la menor influencia posible. Consideramos que la autoría/autonomía se estructura inversamente proporcional a las exigencias burocráticas. A continuación, presentamos el análisis de la frecuencia en los foros de formación del curso investigado.

\section{Sobre la frecuencia en los Foros}

Para este análisis, consideramos tres indicadores substanciales para entender la dialogía en un curso online: 1) La frecuencias con que los agentes (educadores y aprendices) se manifiestan en los foros; 2) La cantidad de mensajes; 3) Los elementos comunicacionales que favorecen la dialogía de los mensajes.

Para el primero y el segundo indicador de la dialogía, analizamos los mensajes de, por lo menos, un foro por clase, constituyendo 109 mensajes de los profesores y 476 de los aprendientes. Observamos que el tiempo de respuesta por parte de los profesores varió de entre 0 a 15 días, con tiempo medio de 3,9 días. El porcentual de mensajes de los profesores que tuvieron secuencia/ debate, en este caso fue considerado cuando por lo menos un alumno se manifestaba a partir del mensaje del profesor, y el porcentual relativo en relación con el tiempo de respuesta de los profesores. En el Cuadro I presentamos los datos con el total de mensajes de los foros analizados. 


\section{I/: Educomunicación mestiza y en movimiento: II: Desafios a un conocimiento multicultural}

Tabla: Mensagens analisadas

\begin{tabular}{|l|l|l|}
\hline $\begin{array}{l}\text { Total de mensajes analiza- } \\
\text { das }=482\end{array}$ & Porcentuales \\
\hline Mensajes dos profesores & 20,0 \\
\hline Tuvieran secuencia/debate & 42,1 \\
\hline Respuestas en el mismo día & 22,9 & 16,7 \\
\hline Respuesta en 1 día & 14,6 & 7,3 \\
\hline Respuesta en 2 días & 6,3 & 3,1 \\
\hline Respuesta en 3 días & 5,2 & 3,1 \\
\hline
\end{tabular}

En el Cuadro I podemos percibir un declive significativo en la medida en que aumenta el tiempo de respuesta de los profesores. Se nota que, cuando los profesores responden en el mismo día, la posibilidad de haber continuidad de la respuesta es mayor (22.9\%). Cuanto más aumenta el tiempo de respuesta del profesor, más disminuye el porcentual de mensajes que tuvieron continuidad en el debate. Apenas 16,7\% de los mensajes de los profesores ocurrieron en el mismo día en que ocurrieran los mensajes de los aprendices. Sobre el primer indicador, nos llamó la atención por la media porcentual de mensajes de los profesores que consideramos relativamente baja (20\%). Además de esto, en apenas $18 \%$ de los casos, el profesor retornó al debate. Otro dato preocupante fue la media de los mensajes entre los profesores, relativamente alta (3,9 días). La variación fue de hasta 15 días para el envío de respuesta, habiendo caso en que el profesor respondió a todos en un único día en un foro que prolongó 18 días.

Aunque no hayamos encontrado en la literatura ningún estudio que indique el tiempo ideal de respuesta del profesor, este trabajo indica que cuando la respuesta del profesor sucede en el mismo día, se tiene una mayor oportunidad de tener continuidad en el debate. Sin embargo, no es el único indicador y volveremos a tratar de esta cuestión más adelante.

Es comprensible esta tendencia, visto que, aunque sea una actividad asíncrona, el propio diálogo, a espera por la manifestación de los interlocutores. Como dice Paulo Freire, "la educación se constituye en un acto colectivo, solidario, un intercambio de experiencias, en que cada sujeto discute sus ideas y concepciones" (Freire, 1998: 96). Aunque el proyecto del curso indicase que el profesor debería retornar las demandas/ preguntas de los estudiantes en un máximo 24 horas, lo que se observa en esta tabla en cuestión, bien como en el ambiente del curso, cierta displicencia en el acompañamiento de las discusiones por el tiempo entre una participación y otra de los profesores/mediadores.

Consideramos que, no habiendo la preocupación, por parte del profesor, en acompañar/participar/mediar el diálogo formativo esperado en foros destinados a este fin, queda perjudicado.

\section{Sobre los niveles de dialogía}

Analizamos los niveles de dialogía a partir de los contenidos en el contexto de cada discusión a tenor de la comprensión de que no hay como hablar de dialogía en textos de mensajes aislados. Es en el corolario de los mensajes en un foro con una temática definida y un mediador reconocido por los participantes, que cada mensaje se convierte parte de una narrativa escrita por todos los participantes.

Los mensajes fueron categorizados en niveles de dialogía a partir de los siguientes criterios: A) Nivel alto - los mensajes propositivos que contenían cues- 


\section{II: Educación, Comunicación y Cultura Contemporánea}

tionamiento con el objetivo de provocar el debate. Una reflexión integrada, contextualizada en el debate o apenas una pregunta con la finalidad de provocar el diálogo. B) Nivel medio - los mensajes que, aunque estuvieran conectados al contenido del foro. El autor no cuestiona y ni retorna al tema en la misma discusión. Apenas una reflexión/opinión. C) Nivel bajo - mensajes que atendiesen la exigencia burocrática del curso, tal como una síntesis de lectura, sin la preocupación de la continuidad del mensaje anterior. No retorna el eventual comentario. O hasta fuera de contexto, que configura como una "conversación paralela" no tratándose del tema en debate.

Con esta categorización (Niveles de dialogía) llegamos a los siguientes porcentuales:

Gráfico: Niveles de dialogía

Los datos demostraron que la estrategia dialógica de los profesores se asemeja a la de los alumnos, como observamos en el gráfico de arriba. Las líneas tienen una tendencia parecida. Aproximadamente $80 \%$ de los mensajes reportan a los aprendices. De estos, 42,1\% de los mensajes de los discentes atendían al apelo para la participación en los foros. Son mensajes que tenían un contenido consistente con la discusión, sin embargo, no participaban del corolario de significados. Son citaciones de textos o manifestaciones de opinión. Como si fuese en una sala con diversas personas discutiendo un determinado asunto, alguien abriese la puerta, dijese alguna cosa y saliese sin importarle la opinión de los otros sobre su manifestación. Aunque los estudiantes no tuviesen el papel de mantener el nivel alto de dialogía, consideramos muy alto el porcentual de mensajes con bajo nivel de dialogía.

Sobre la participación de los profesores fue esperado un nivel alto de dialogía en los mensajes, pues al final eran ellos quienes tenían la función de la mediación/ animación de los foros. Pero, en esta investigación identificamos $43,5 \%$ de los mensajes de los profesores con bajo nivel de dialogía. Apenas 13,9\% fueron mensajes propositivos que, de hecho, cumplían su papel de promover el diálogo. Es decir, había un contenido significativo y cuestionamientos que contribuían al debate. Son mensajes que ayudaban para que el foro no se convirtiese en un conjunto de "retales" de opiniones que no substancian el aprendizaje. Es papel de los profesores conducir la discusión en el foro para que haya un debate propositivo y que los significados de cada mensaje sean valorizados.

Veamos el caso siguiente en que presentamos en secuencia el mensaje de un alumno del foro y el mensaje del profesor. Este hizo una afirmación genérica sin encadenamiento con el mensaje anterior y clasificamos de bajo nivel de dialogía. En seguida el mediador hizo su cuestionamiento e indicó el camino para encontrar base teórica para la manifestación, lo que categorizamos como de alto nivel de dialogía.

Re: VAMOS A HABLAR SOBRE EL PPP (Proyecto

Político-Pedagógico de un centro educacional)

Por (Aprendiz 1) - lunes 27 abril 2015, 11:57

El PPP de la escuela en que trabajo, define la identidad de la U.E (Unidad Educacional) indica caminos para una enseñanza de calidad.

Re: VAMOS A HABLAR SOBRE EL PPP

Por (Profesor) - miércoles, 6 mayo 2015, 22:17

Me gustaría que usted explicase cómo es la construcción, y cómo son construidos esos caminos para la enseñanza de calidad. Los textos de la biblioteca ayudaran bastante a sus reflexiones.

Notemos que el mensaje del profesor/mediador es publicada 10 días después del post del aprendiz y no 


\section{II: Educomunicación mestiza y en movimiento: 11. Desafios a un conocimiento multicultural}

tiene secuencia. En este caso, ni él vuelve para pedir del estudiante una profundización en el debate. Mismo así, si tomamos el mensaje, aislado de su contexto, podemos decir que el mensaje del profesor tuvo una presencia social y pedagógica (Garrison, Anderson y Archer, 2001).

Veamos la secuencia de mensajes en que el profesor/ mediador no ejerce su papel de mediador.

Re: COMUNICACIÓN EN LA ESCUELA

Por (Aprendiz 2) - lunes 12 octubre 2015, 16:41

Creo que la escuela debe adaptarse a la cultura mediática y usarla como aliada para el progreso de la educación, pues ignorarla significa "tapar los ojos" para un asunto que está en todos los espacios (...) en los pasillos y en los patios de las escuelas nos deparamos con alumnos interconectados, (...) Así, viene la indagación: ¿Por qué es tan difícil usar las tecnologías de la información y comunicación como herramienta en el aula? Usamos las TICs en nuestro cotidiano, entonces, ¿Cuál es el obstáculo para usar ese conocimiento envolviendo los contenidos que serán tratados en la clase? Sé que existen varios desafíos (...). Pero como tenemos que hacer nuestra parte, sería interesante colocar esos asuntos para discusión y reflexión en nuestras escuelas.

Re: COMUNICACIÓN EN LA ESCUELA

Por (Aprendiente 3) - lunes 19 octubre 2015, 17:45

Estoy de acuerdo con usted, realmente debemos reflexionar sobre eso.

Re: COMUNICACIÓN EN LA ESCUELA

Por (Profesor) - miércoles 21 octubre 2015, 10:28

Veo que la mayoría de las escuelas no están preparadas para lidiar con la temática en la clase, por eso que la situación se está convirtiendo en indisciplina en vez de contribuir con el proceso de enseñanza y aprendizaje.

En el primer mensaje, el aprendiz hace una óptima reflexión y la clasificamos de alto nivel de dialogía. Siete días después otro estudiante apenas concuerda con el otro mensaje, la cual clasificamos de baja dialogía. El profesor mediador entró en esa conversación nueve días después del primer mensaje. Considerado mucho tiempo en aquel contexto. Además del tiempo, el profesor hizo un comentario síntesis, con fuerte significado negativo. La forma que él se manifiesta no estimula la continuidad de la discusión, lo que consideramos de bajo nivel de dialogía.

En esta investigación encontramos una situación inusitada: en un grupo en que los profesores estaban muy presente en los debates, ocurrió una discusión temática con 133 mensajes, sin la participación del mediador. Sin embargo, en ese grupo hubo un trabajo intensivo de interacción con los estudiantes. Fueron abiertos más de 50 foros durante el curso. Mientras que lo esperado sería de 10 foros por grupo. Hubo más de tres mil mensajes durante el curso, mientras que el grupo con menos interacción llegó a apenas un $10 \%$ de ese total. Lo que nos lleva a afirmar que en el contexto del curso, la riqueza de la dialogía/autoría puede ser determinante en la motivación y el éxito de los foros.

\section{Posibles conclusiones}

La dialogía en los cursos, en especial en los foros de los cursos online, es un desafío de nuestra cultura educacional y, apenas, diferente a la dificultad que se tiene en los cursos tradicionales (presenciales). Una de las diferencias importantes es que, en los cursos virtuales, queda el registro, lo que facilita la percepción de las dificultades entre los estudiantes y educadores en el diálogo pedagógico. 


\section{II: Educación, Comunicación y Cultura Contemporánea}

Como vimos, no es suficiente la participación para que la dialogía acontezca, es necesario que el mediador entienda tanto el contenido como la metodología para que éste no desanime un foro con mensajes de bajo nivel dialógico.

Se sugiere el uso de estrategias de discurso que personalice el diálogo, tales como llamar por el nombre, elogiar, llamar la atención de forma delicada, invitar a un participante a analizar un mensaje o a responder la pregunta de otro, percibir silencios de algún participante y llamarlo para participar, etc.

Otro aspecto de la dialogía que nos llamó la atención fue la importancia del contexto dialógico del foro. Entendemos la presencia virtual permanente de los mediadores, principalmente en las primeras 24 horas después del mensaje ser publicado por el aprendiz, de fundamental importancia para que el foro tenga su continuidad en el debate.

Entendemos que no hay forma de cómo estimar con precisión cuál es la proporción adecuada entre los mensajes envolviendo estudiantes y profesores, pero consideramos el sentido común pedagógico. Será la experiencia del profesor la capaz de indicar el límite y la calidad de la mediación. Vimos un curso en que consiguen mantener sus aprendices interactuando permanentemente, puede haber debates que el profesor pueda tener una participación mínima.

Consideramos la frecuencia tan importante como los demás indicadores, por ser condición para que los aprendices perciban la presencia y mantengan el vínculo psicológico con la comunidad de aprendizaje, que Moore (1993) llamó de "distancia transaccional". La pericia y el sentido común de los mediadores debe indicar una relación inversa a sus intervenciones: cuanto más animado y con mensajes de alto nivel de dialogía, menos deben intervenir los mediadores en el debate.

¿Es posible estructurar un curso a partir del diálogo? Pensamos que sí. Sin embargo, es preciso que los participantes: educadores y aprendientes, hagan de él un medio provocador en la gestión del aprendizaje. En este caso, será preciso mucha atención de los educadores en la forma pedagógica y hacer del ambiente del curso un espacio virtual de encuentro de una comunidad de aprendizaje que, colaborativamente, ejerza la gestión del aprendizaje.

Notas

(1) Las citas fueron traducidas

\section{Referencias}

Bakhtin, M. M. (1997). Estética da criação verbal (2 ed.). (a. E. Pereira, Trad.) São Paulo: Martins Fontes. Bardin, L. (2011). Análise de conteúdo. Lisboa: Edições 70.

Flick, U. (2005). Método qualitativos na investigação científica. Lisboa: Monitor.

Freire, P. (1975). Extensão ou comunicação? Rio de Janeiro: Paz e Terra.

Freire, P. (1979). Conscientização: teoria e prática da libertação. São Paulo: Cortez \& Moraes.

Garrison, D., Anderson, T. y Archer, W. (2001). Critical Thinking, Cognitive Presence, and Computer Conferencing in Distance Education. The American Journal of Distance Educational, 1(15); 7-23. Disponible en: http://cde.athabascau.ca/coi_site/documents/ Garrison_Anderson_Archer_Critical_Inquiry_model. pdf. Acesso em abril de 2016. 


\section{I/: Educomunicación mestiza y en movimiento: 1. Desafios a un conocimiento multicultural}

Litto, F. M. y Formiga, M. M. (Eds.). (2009). Educação a distância : o estado da arte. São Paulo: Pearson Education do Brasil.

Martins, J. L. (2014). A gestão da aprendizagem em ambiente virtual. Tese de doutoramento, Universidade do Minho, Instituto de Educação, Braga; Portugal. .
Scorsolini-Comin, F. (2014). Diálogo e dialogismo em Mikhail Bakhtin e Paulo Freire: contribuições para a educação a distância. Educação em Revista, $30(3) ; 245-256$. 